\title{
Convenient Contrast Enhancement by Hole Free Phase Plate in a TEM
}

\author{
Marek Malac*, Masahiro Kawasaki”, Marco Beleggia`, Peng Li", Ray Egerton* \\ *National Institute of Nanotechnology, 11421 Saskatchewan Drive, Edmonton, Canada. \\ \# JEOL USA, 11 Dearborn Road, Peabody, MA, 01960 \\ •Center for Electron Nanoscopy, Technical University of Denmark, Lyngby, Denmark.
}

Minimizing the electron irradiation dose is crucial for radiation sensitive (biological) samples. A (Zernike) phase plate (PP) in a TEM reduces the irradiation dose needed to obtain a desired signal to noise ratio (SNR) [1]. This is achieved mainly by efficient transfer of low spatial frequencies that have large contribution to intensity in phase imaging.

The charging of objects irradiated by an electron beam is well known effect [2] and in case of a PP it was described, in particular, by Danev et al. [3]. We have studied thin-film type phase plates (PP) [3] made from various materials (amorphous carbon, chromium-carbon multilayers, aluminum and gold) in a JEOL 2200 FS. We observed modification of the contrast transfer function (CTF) and attribute this to charging of the PP. Often, the CTF is dominated by charging rather than the $\pi / 2$ phase shift of the thin-film itself. Fig. 1 a) schematically depicts a PP cross section and $b$ ) a view along the beam direction. Although the processes that determine the level of charging are not understood, it is likely that the SE emission is the reason for PP charge buildup. Charge buildup implies existence of an electric field that in turn affects the CTF through the associated electrostatic potential. Depending on the SE yield, conductivity of the PP film and of a surface contamination of the PP, a sufficient potential can develop on the PP and govern the the CTF. It should be noted that even in conductors, a small but non-zero electric field must be present to drive a current compensating for the SE emission. SE emission is present in all materials as evidenced by the ability to obtain SE images in SEM and scanning TEM.

Fig. 2 b) shows a CTF for ZPP (a $\pi / 2$ thick carbon PP with a hole for central beam). Although the defocus was unchanged between Fig. 2a) and b), the change in CTF does not correspond to simple $\pi / 2$ shift due to ZPP insertion. Our simulations suggest that shape of the $\mathrm{CTF}$ and the band indicated by an arrow in b) can be explained by PP charging. Since the CTF is affected by charging, a question arises whether a hole-free PP can be used to improve transfer of low frequencies in a phase TEM. Fig. 3 a) shows an image of elk fibrils acquired with no phase plate in the beam path, b) with amorphous carbon PP without a hole in the beam path; and c) with crystalline gold film PP without hole. We attribute the contrast transfer in b) and c) mainly to charging of the PP. Figs. b) and c) exhibit about 2 to 3 times higher contrast than a). Fig. 4 shows the CTF for Fig 3 a), b) and c) respectively. A transfer band at low spatial frequencies, marked by an arrow, broader in b) and c) than in a). Real-time observations show that the CTF in a PP or Zernike PP changes with irradiation time and reaches a steady state in 1 min to $15 \mathrm{~min}$, depending on PP material, possibly PP contamination and on beam condition.

Thickness maps of the PPs after extensive use exclude excessive contamination as a factor solely determining the CTF, but a thin contamination layer can not be excluded. The possibility of using a PP without a hole significantly simplifies PP preparation, eliminates the need for precision alignment of a PP and simplifies mounting the PP in the microscope beam path [5,6]. Alternatively a hole in PP (resulting in traditional ZPP) can be fabricated in-situ using electron-beam-induced etching and sputtering [5], again eliminating the need for a precision alignment mechanism for the ZPP hardware. A potential advantage of self-charging PP is that 
the PP thickness can be very small (as permitted by mechanical properties of the used material). Small PP thickness the leads to low inelastic scattering in the PP film itself further improving contrast transfer efficiency.

\section{References:}

[1] Malac M, Beleggia M, Egerton RF, Zhu Y, Ultramicroscopy 108 (2008), 126.

[2] Reimer L, Transmission Electron Microscopy, $4^{\text {th }}$ edition, Springer 1997.

[3] Danev R, Glaeser RM, Nagayama K, Ultramicroscopy,109 (2009), 312.

[4] Egerton RF et. al., Micron, 35, (2004), .399.

[5] Malac M et. al., patent submitted (2009).

[6] Malac et al. Microscopy \& Microanalysis, Vol 15 (2009), Suppl.2, p. 1234.

[7] The support for this work was provided by NINT/NRC and by JEOL USA (M.K.). The elk fibril samples were provided by Prof. Howard Young (NINT), CNT sample was provided by F. Paraguay-Delgado.

a)

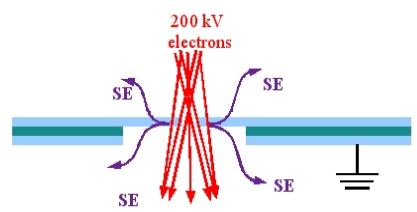

b)

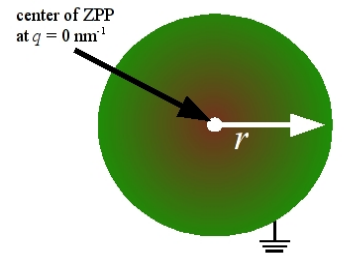

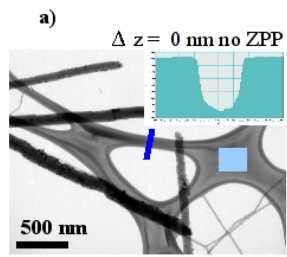

c)

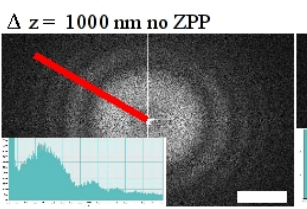

b) $\Delta \mathrm{z}=0 \mathrm{~nm}$ with $\mathrm{ZPP}$

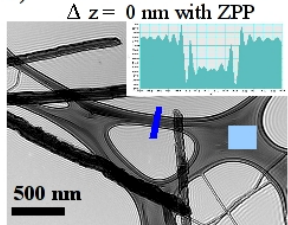

$\Delta \mathrm{z}=0 \mathrm{~nm} \mathrm{ZPP}$ inserted

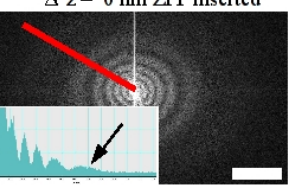

Fig. 1a) cross sectional view of a PP. A multilayer $(\mathrm{C}+\mathrm{Cr}+\mathrm{C}$ layers, $1 / 2 \pi$ each $)$ is depicted. $\mathrm{SE}$ emission is indicated by the curved arrows while incident beam is indicated by straight arrows. b) view of a PP with a central hole (Zernike PP) along the beam direction.

a)

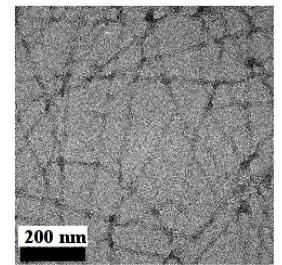

b)

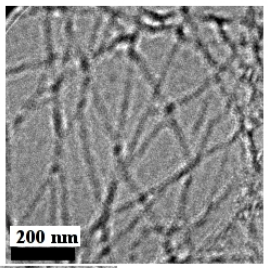

c)

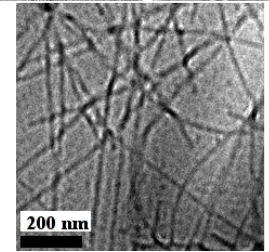

Fig. 3 a) Elk fibril imaged with no phase plate in the beam path, only defocus was applied. With carbon phase plate without hole in the beam path b); and c) with gold film phase plate without hole. We attribute the contrast transfer in b) and c) mainly to charging of the thin film phase plate in the back focal plane. The contrast improve by about a factor 2 to 3 in b) and c) compared to a).

Fig 2. a) and c) image acquired without ZPP at defocus marked in image. b) and c) with thin film carbon ZPP with a hole with cut on frequency of $0.04 \mathrm{~nm}^{-1}$ and zero defocus. The transfer band marked by an arrow in d) arises due to charging of the ZPP.

a)

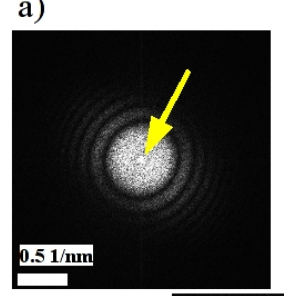

b)

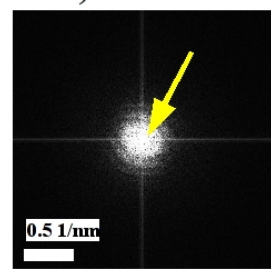

c)

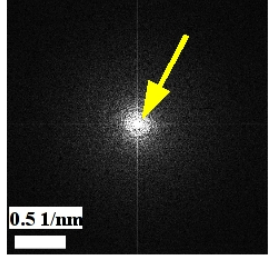

Fig. 4 CTF obtained using a) objective lens defocus only, b) carbon phase plate without a hole, c) gold phase plate without hole. In all cases the transfer band near low spatial frequencies is marked by an arrow. In b) and c) the transfer band is broad and much closer to $q=0 \mathrm{~nm}^{-1}$ than in a) resulting in efficient transfer of very low spatial frequencies. 